\title{
CONGRUENCE PERMUTABLE EXTENSIONS OF DISTRIBUTIVE DOUBLE $p$-ALGEBRAS*
}

\author{
by R. BEAZER and J. SICHLER
}

(Received 20th October 1991)

\begin{abstract}
Every distributive double $p$-algebra $L$ is shown to have a congruence permutable extension $K$ such that every congruence of $L$ has a unique extension to $K$.
\end{abstract}

1990 Mathematics subject classifications: Primary 06D15; Secondary 08A30.

\section{Introduction}

It is well-known that any distributive lattice $L$ can be embedded into a distributive relatively complemented latice $K$ in such a way that every congruence of $L$ has exactly one extension to $K$ (see [4]). Furthermore, Katrinák [5] has shown that any distributive $p$-algebra $L$ can be embedded into a distributive $p$-algebra $K$ whose dense filter is relatively complemented in such a way that every congruence of $L$ has exactly one extension to $K$. However, a distributive lattice is congruence permitable if and only if it is relatively complemented and Berman [2] has shown that a distributive $p$-algebra is congruence permutable if and only if its dense filter is relatively complemented. Consequently, every distributive lattice $L$ and every distributive $p$-algebra $L$ has a congruence permutable extension $K$ such that every congruence of $L$ has exactly one extension to $K$. In this note we obtain an analogous result for distributive double $p$ algebras, using Priestley duality.

\section{Preliminaries}

Although we assume some acquaintance with Priestley duality for distributive $(0,1)$-lattices, we begin by reviewing some notation and terminology and the restriction of Priestley duality to distributive double $p$-algebras.

Let $(X, \tau, \leqq)$ be an ordered topological space and let $Y \subseteq X$. The set $Y$ is said to be decreasing (increasing) if $(Y]_{X}=Y\left([Y)_{X}=Y\right)$, where

$$
(Y]_{X}=\{x \in X: x \leqq y \text { for some } y \in Y\}
$$

and $[Y)_{X}$ is defined dually.

Both authors gratefully acknowledge the support of the NSERC. 
We will write $\operatorname{Max}_{X}(Y)$ for $[Y)_{X} \cap \operatorname{Max}(X)$ and $\operatorname{Min}_{X}(Y)$ for $(Y]_{X} \cap \operatorname{Min}(X)$, where $\operatorname{Max}(X)$ and $\operatorname{Min}(X)$ denote the set of maximal elements and minimal elements of $X$, respectively. We also define $\operatorname{Ext}_{X}(Y)=\operatorname{Max}_{X}(Y) \cup \operatorname{Min}_{X}(Y), \quad \operatorname{Ext}(X)=\operatorname{Ext}_{X}(X)$, $\operatorname{Mid}(X)=X \backslash \operatorname{Ext}(X)$ and, when $Y=\{x\}$, we write $\operatorname{Max}_{X}(x)$ for $\operatorname{Max}_{X}(Y)$ and $\operatorname{Min}_{X}(x)$ for $\operatorname{Min}_{X}(Y)$.

$(X, \tau, \leqq)$ is called a Priestley space if it is compact and totally order disconnected; in the sense that, for every $x, y \in X$ with $x \leq y$, there exists a clopen decreasing set $Y \subseteq X$ such that $y \in Y$ and $x \notin Y$. In such spaces, the sets $\operatorname{Max}_{X}(x)$ and $\operatorname{Min}_{X}(x)$ are non-empty, for any $x \in X$, and the following separation property holds:

(s) For any closed $Y, Z \subseteq X$ with $Y \cap(Z]_{X}=\phi$ there exists a clopen decreasing set $D$ such that $(Z]_{X} \subseteq D$ and $Y \cap D=\phi$.

If $\mathscr{P}$ is the category of all Priestley spaces and continuous order preserving mappings and $\mathscr{D}$ is the category of all distributive $(0,1)$-lattices and $(0,1)$-lattice homomorphisms then Priestley $([6,7])$ has shown that there exist contravariant functors $D$ and $P$ from $\mathscr{P}$ into $\mathscr{D}$ and $\mathscr{D}$ into $\mathscr{P}$, respectively, such that the composite functors $P \circ D$ and $D \circ P$ are naturally equivalent to the identity functors on their domains. Furthermore, a morphism $f$ in $\mathscr{P}$ is subjective if and only if $D(f)$ is an embedding.

Recall now that a distributive double p-algebra is an algebra $(L ; \vee, \wedge, *,+, 0,1)$ in which $(L ; \vee, \wedge, 0,1)$ is a distributive $(0,1)$-lattice and, for $a \in L, a^{*}$ is characterized by $x \leqq a^{*} \Leftrightarrow a \wedge x=0$ and $a^{+}$is characterized in a dual fashion. Priestley [8] has described the duals of distributive double $p$-algebras as follows:

(1) For an object $\mathbf{X}=(X, \tau, \leqq)$ in $\mathscr{P}, D(X)$ is a double $p$-algebra if and only if $[Y)_{X}$ is clopen for every clopen decreasing set $Y \subseteq X$ and $(Y]_{X}$ is clopen for every clopen increasing set $Y \subseteq X$.

(2) For a morphism $f:(X, \tau, \leqq) \rightarrow\left(X^{\prime}, \tau^{\prime}, \leqq^{\prime}\right)$ in $\mathscr{P}, D(f)$ is a double p-algebra homomorphism if and only if $f\left(\operatorname{Max}_{X}(x)\right)=\operatorname{Max}_{X^{\prime}}(f(x))$ and $f\left(\operatorname{Min}_{X}(x)\right)=$ $\operatorname{Min}_{X^{\prime}}(f(x))$, for every $x \in X$.

If $D(X)$ is a double $p$-algebra then $\mathbf{X}$ is called a dp-space and in such spaces $\operatorname{Max}(X)$ and $\operatorname{Min}(X)$ are closed. If $D(f)$ is a double $p$-algebra homomorphism than $f$ is called a dp-map.

Finally, we recall from [3], the following facts. If $\mathbf{X}=(X, \tau, \leqq)$ is a $d p$-space, $L=D(\mathbf{X})$ and $Y$ is a closed c-set, i.e. $Y$ is a closed subset of $X$ satisfying $\operatorname{Ext}_{X}(Y) \subseteq Y$, then the binary relation $\Theta_{L}(Y)$ defined on $L$ by

$$
U \equiv V\left(\Theta_{L}(Y)\right) \Leftrightarrow U \cap Y=V \cap Y
$$

is a congruence and the map $Y \mapsto \Theta_{L}(Y)$ is a 1-1 correspondence between the lattice of closed $c$-sets of $\mathbf{X}$ and the congruence lattice of the distributive double $p$-algebra $L$.

\section{The construction}

Our decision to employ Priestley duality to achieve our goal was motivated partly by the following result. 
A distributive double p-algrbra has permutable congruences if and only if there is no 4-element chain in its dp-space.

This and other characterizations of congruence permutable distributive double $p$ algebras may be found in [1].

Theorem. Every distributive double p-algebra $L$ has a congruence permutable extension $K$ such that every congruence of $L$ has exactly one extension to $K$.

Proof. Let $P=(X, \tau, \leqq)$ be the Priestley dual of the distributive double $p$-algebra $L$ and let $Q=(X, \tau, \preceq)$ where $\preceq$ is the binary relation defined on $X$ by

$$
u \preceq v \Leftrightarrow\{u, v\} \cap \operatorname{Ext}(P) \neq \phi \text { and } u \leqq v .
$$

Clearly $\preceq$ is a partial ordering of $X, \operatorname{Max}(Q)=\operatorname{Max}(P)$ and $\operatorname{Min}(Q)=\operatorname{Min}(P)$, so that $\operatorname{Ext}(Q)=\operatorname{Ext}(P)$. Furthermore, $\operatorname{Mid}(Q)$ is an unordered copy of $\operatorname{Mid}(P), \operatorname{Max}_{Q}(x)=$ $\operatorname{Max}_{P}(x)$ and $\operatorname{Min}_{Q}(x)=\operatorname{Min}_{P}(x)$, for every $x \in X$. Observe that there is no 4-element chain in $Q$ and that the identity mapping $f: Q \rightarrow P$ is continuous, preserves order and, subject only to our showing that $Q$ is a $d p$-space, has the properties necessary for it to qualify as a $d p$-map. We proceed by showing that $Q$ is, indeed, a $d p$-space. Clearly, $Q$ is compact. With the intention of proving that $Q$ is totally order disconnected, suppose that $x, y \in X$ and $x \npreceq y$.

Let us assume that $x \leqq y$. Then there exists a clopen $\leqq$-decreasing set $C \subseteq X$ such that $y \in C$ and $x \notin C$. However, the set $C=f^{-1}(C)$ is clopen and $\preceq$-decreasing because $f$ is continuous and order preserving.

In the event that $x \leqq y$ and $x \npreceq y$, we have $x<y$ and it follows from the definition of $\preceq$ that $x, y \in \operatorname{Mid}(Q)=\operatorname{Mid}(P)$. Since $y \notin \operatorname{Max}(P)$, we have $(y]_{P} \cap \operatorname{Max}(P)=\phi$ and so that separation property (s) guarantees the existence of a clopen $\leqq$-decreasing set $A_{0}$ such that $y \in A_{0}$ and $A_{0} \cap \operatorname{Max}(P)=\phi$. Also, since $y \notin \operatorname{Min}(P)$, the closed set $\{x\} \cup \operatorname{Min}(P)$ has empty intersection with $[y)_{P}$ and the dual of the separation property (s) guarantees the existence of a clopen $\leqq$-increasing set $A_{1}$ such that $y \in A_{1}$ and $A_{1} \cap(\{x\} \cup \operatorname{Min}(P))=\phi$. Then set $A=A_{0} \cap A_{1}$ is, therefore, a clopen convex subset of $\operatorname{Mid}(P)$ having the property that $y \in A$ and $x \notin A$. The set $A$ also has these properties with respect to $Q$, since $f$ preserves order and is continuous. Furthermore, since $x \notin \operatorname{Min}(P)$, we can use the separation property (s) to obtain a clopen $\leqq$-decreasing set $B$ such that $x \notin B$ and $\operatorname{Min}(P) \subseteq B$. Again, $B$ is clopen and $\preceq$-decreasing. We claim that $C=A \cup B$ fulfills our needs. Obviously, $C$ is clopen, $y \in C$ and $x \notin C$. To show that $C$ is $\preceq$-decreasing, suppose that $z \preceq c \in C$ and $z \neq c$. If $c \in B$ then $z \in B \subseteq C$, since $B$ is $\preceq$-decreasing, whereas if $c \in A$ then $c \in \operatorname{Mid}(Q)$ and so $z \in \operatorname{Min}(Q) \subseteq B \subseteq C$. Thus, $Q$ is a Priestly space.

Next, we show that $[D)_{Q}$ is clopen, for any clopen $\preceq$-decreasing set $D \subseteq X$. Since $D$ is $\preceq$-decreasing, we have $\operatorname{Min}_{Q}(D)=D \cap \operatorname{Min}(Q)$. Moreover, because $m \preceq x$ is equivalent to $m \leqq x$, for any $m \in \operatorname{Min}(Q)=\operatorname{Min}(P)$, we have $\operatorname{Min}_{P}(D)=\operatorname{Min}_{Q}(D)=D \cap \operatorname{Min}(P)=M$. This relation between the two orders also implies that $[M)_{P}=[M)_{Q}$. However, $[D)_{Q}=$ $[M)_{Q}$, since $D$ is $\preceq$-decreasing. Therefore $[M)_{P}=[D)_{Q}$. Now, $M \subseteq D$ implies that $\{x\} \cap M=\phi$, for any $x \notin D$. Since $M$ is closed and $\leqq$-decreasing, the separation property (s) ensures the existence of a clopen $\leqq$-decreasing set $E_{x}$ such that $M \subseteq E_{x}$ and $x \notin E_{x}$. 
Now, $\cup\left\{X \backslash E_{x}: x \in X \backslash D\right\} \supseteq X \backslash D$ and $X \backslash D$ is compact, since $D$ is open. Therefore there is a finite $F \subseteq X \backslash D$ such that $\cup\left\{X \backslash E_{x}: x \in F\right\} \supseteq X \backslash D$. The set $E=\cap\left\{E_{x}: x \in F\right\}$ is clopen, $\leqq$-decreasing and satisfies $M \subseteq E \subseteq D$. Clearly, $M \subseteq \operatorname{Min}_{P}(E) \subseteq \operatorname{Min}_{P}(D)=M$ so that $\operatorname{Min}_{P}(E)=M$ and therefore $[M)_{P}=[E)_{P}$, since $E$ is $\leqq$-decreasing. It follows now that $[D)_{Q}=[E)_{P}$ which is clopen, since $E$ is clopen and $P$ is a $d p$-space. This, together with a dual argument, completes the proof of the fact that $Q$ is a $d p$-space. Summarizing, thus far, $K=D(Q)$ is a congruence permutable extension of $L$.

Finally, observe that a subset of $X$ is a closed $c$-set in $P$ if and only if it a closed $c$ set in $Q$, since ordered pairs involving extremal elements are the same in either $d p$-space, and so $\Theta_{K}(C)$ is the unique extension of $\Theta_{L}(C)$ to $K$, for any clopen $c$-set $C$.

Corollary. The congruence lattice of any distributive double p-algebra is isomorphic to the congruence lattice of some congruence permutable distributive double p-algebra.

Concluding remarks. Recall that an algebra is congruence regular if each of its congruences is uniquely determined by any one of its classes. Varlet [9] has shown that the congruence regular distributive double $p$-algebras are precisely those having no 3element chain in their $d p$-spaces. It is an open question as to whether or not the congruence lattice of an arbitrary distributive double $p$-algebra is isomorphic to the congruence lattice of some congruence regular distributive double $p$-algebra.

\section{REFERENCES}

1. M. E. Adams and R. Beazer, Congruence properties of distributive double p-algebras, Czechoslovak Math. J. 41 (1991), 216-231.

2. J. Berman, Congruence relations of pseudocomplemented distributive lattices, Algebra Universalis 3 (1973), 288-293.

3. B. A. Daver, Subdirectly irreducible distributive double p-algebras, Algebra Universalis 8 (1978), 73-88.

4. G. Grätzer, Lattice Theory: First Concepts and Distributive Lattices (Freeman, San Francisco, California, 1971).

5. T. KatrIŃÁK, Congruence lattices of distributive p-algebras, Algebra Universalis 7 (1977), 265-271.

6. H. A. Priestley, Representation of distributive lattices by means of ordered Stone spaces, Bull. London Math. Soc. 2 (1970), 186-190.

7. H. A. Priestley, Ordered topological spaces and the representation of distributive lattices, Proc. London Math. Soc. 24 (1972), 507-530.

8. H. A. Priestley, Ordered sets and duality for distributive lattices, Ann. Discrete Math. 23 (1984), 36-90.

9. J. VARLET, A regular variety of type $\langle 2,2,1,1,0,0\rangle$, Algebra Universalis 2 (1972), 218-223.

\author{
Department of Mathematics \\ UNIVERSITY OF GLASGOW \\ Glasgow \\ G12 8QW \\ SCOTLAND
}

Department of Mathematics

UNIVERSITY OF MANITOBA

Winnipeg, Manitoba

CANAda

R3T 2N2 